\title{
Syndrome of absent abdominal muscles: two cases with microcephaly, polymicrogyria, and cerebellar malformations
}

\author{
REID R. HEFFNER ${ }^{1}$ \\ From the Department of Pathology, Yale University School of Medicine, New Haven, \\ Connecticut, U.S.A.
}

SUMMARY Two unique cases of the syndrome of absent abdominal muscles with central nervou system involvement are presented. Microcephaly, polymicrogyria, and cerebellar heterotopiae were present in both. In case 1 there was also absence of the corpus callosum and agenesis of the cerebellap vermis. In case 2 a count of anterior horn cells in the spinal cord showed a reduction of approxi mately $50 \%$ in the lower thoracic region. The pertinent literature is briefly discussed. The findings in the nervous system suggest that the syndrome is the result of defective embryogenesis during the first trimester.

Congenital absence of the abdominal muscles was first described by Fröhlich in 1839 (Bourne and Cerny, 1967), but not until the turn of the century (Parker, 1895) was this finding known to be associated with genitourinary abnormalities. Eagle and Barrett (1950), who reviewed 42 cases in the world literature and added nine of their own, were among the first to indicate that the deficiency of abdominal muscles is actually part of a syndrome, usually occurring in males, which consists of a triad of abdominal muscle aplasia, massive bladder and ureteral dilatation, and cryptorchism. The kidneys often are affected by secondary hydronephrosis (Silverman and Huang, 1950) or by polycystic dysplasia (Nunn and Stephens, 1961). Commonly associated anomalies include lower urinary tract obstruction, patent urachus, pigeon breast, and talipes. Dislocation of the hip (Metrick, Brown, and Rosenblum, 1957), pulmonary atresia (Brierre, 1963), choanal atresia (Brierre, 1963), and abnormalities of the gastrointestinal and cardiovascular systems (Metrick, Brown, and Rosenblum, 1957) have been reported. Since the original observations by Parker (1895), over 120 cases have been described (Williams and Burkholder, 1967), yet investigations

'Present address (including reprint requests): Section of Neuropathology, Department of Pathology, New York Hospital-Cornell Medical Center, 1300 York Avenue, New York City, New York, 10021, U.S.A. of neurological interest have been few. This rep@irt concerns two cases of the syndrome of absento abdominal muscles with involvement of the centwa nervous system.

CASE 1

This 1 day old male was transferred from a neighbouring hospital to the Yale-New Haven Medical Center for evaluation of absent abdominal muscles. The baby's mother, aged 26, had produced two normal children? previously. The present pregnancy and delivery had beerth uneventful. The gestational age was 37 weeks. Or admission the infant weighed $3,460 \mathrm{~g}$ and measured 50 $\mathrm{cm}$ in crown-heel length. The head was quite small $\vec{D}$ measuring only $30 \mathrm{~cm}$ in circumference, as compared $B$ with a chest circumference of $34 \mathrm{~cm}$. The ears were lows set and the vertex of the skull was flat, giving the forehead a receding appearance. The neck was extremely short. high-arched palate was noted. The abdomen was dis tended due to a lack of abdominal muscles. A patent? urachus was present. The testes were undescendedo Bilateral talipes equinovarus and a dislocated left hip. were evident. Neurological examination disclose $\subseteq$ generalized hypotonia and diminished suck and grasp reflexes. Intravenous pyelography demonstrated pooro visualization bilaterally. Markedly dilated and tortuous ureters were seen on retrograde pyelography. A righ nephrostomy was performed. Post-operatively the patient had several generalized grand mal convulsions for which he was given phenobarbitone. At the age of 7 days the child suffered a fatal cardiorespiratory arrest. 
Necropsy revealed bilateral pleural effusions and focal pulmonary atelectasis. Multiple sections through the anterior abdominal wall failed to yield any gross or histological evidence of the rectus abdominis, external or internal oblique, or transversus abdominis muscles. The muscles of the back and thorax including the intercostals were normal. The kidneys were not enlarged but showed multicystic dysplasia microscopically. The dilated ureters entered the bladder normally. Ganglion cells were numerous in the ureters and bladder. No evidence of lower urinary tract obstruction was found. The testes were located in the abdominal cavity lying superior to the ureters at the ureterovesical junction.

After formalin fixation for 10 days the brain was examined. For the size of the infant, it was small, weighing $220 \mathrm{~g}$. There was polymicrogyria of the medial portions of the frontal and parietal lobes, imparting a Morocco-leather appearance to the superior frontal gyrus and the paracentral lobule. Coronal sections of the cerebral hemispheres revealed absence of the corpus callosum (Fig. 1). The septum pellucidum and fornix were displaced laterally. The anterior commissure and cingulate gyrus appeared normal. The bundle of Probst was prominent. There was apparent dilatation of the third ventricle due to the absent corpus callosum and displaced related structures and actual dilatation of the occipital horns of the lateral ventricles. The posterior inferior cerebellar vermis was absent. From the posterior surface of the cerebellum a direct view could be obtained of the floor of the deep, trough-shaped caudal fourth ventricle, which was covered only by an opaque, thickened pia-arachnoid membrane. Serial horizontal sections demonstrated partial agenesis of the vermis, sparing the flocculus, anterior lobe (Fig. 2), and cerebellar hemispheres. Absence of the declive, folium, tuber, pyramis, uvula, and nodulus resulted in striking anterior-posterior elongation of the caudal fourth ventricle (Fig. 3). The brain-stem was grossly normal. Sections for microscopy

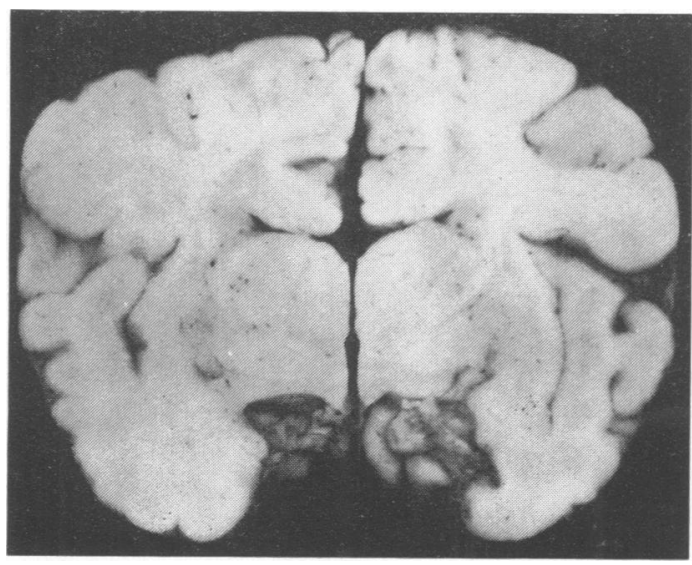

FIG. 1. Coronal section of cerebrum showing absence of corpus callosum. Note bundle of Probst at superior lateral lip of frontal horn.

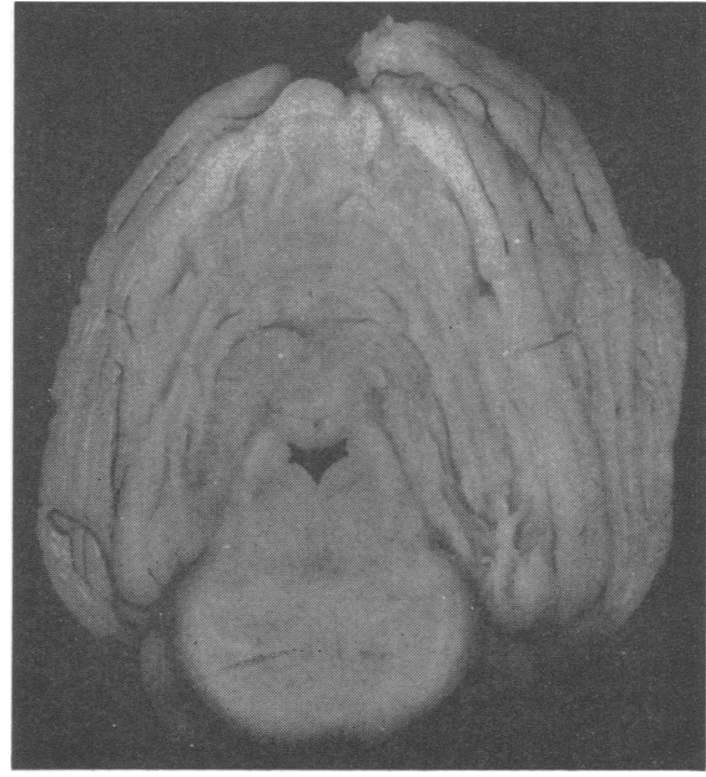

FIG. 2. Horizontal section of cerebellum and rostral pons. Anterior lobe of cerebellum is normal.

were obtained from the frontal, temporal, parietal, and occipital lobes, Ammon's horn, basal ganglia, thalamus, midbrain, pons, medulla, and cerebellum. They were embedded in paraffin, cut at $6 \mu$, and stained with haematoxylin and eosin, Nissl (thionin), and for myelin (Weil). The glandular gyral pattern of polymicrogyria was observed in specimens from the mesial frontal and parietal lobes (Fig. 4). A simplified, three-layer micro-

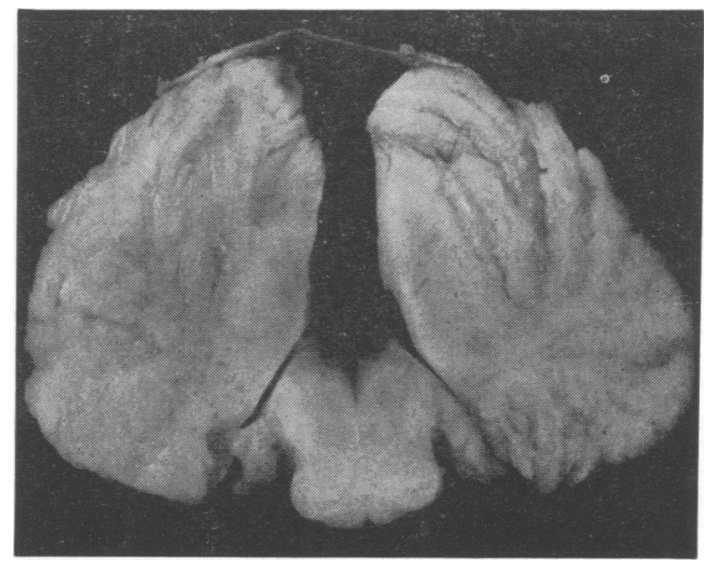

FIG. 3. Horizontal section of cerebellum and medulla. Reconstructed specimen shows absence of cerebellar vermis, producing trough-shaped caudal fourth ventricle which is elongated in anterior-posterior plane. 


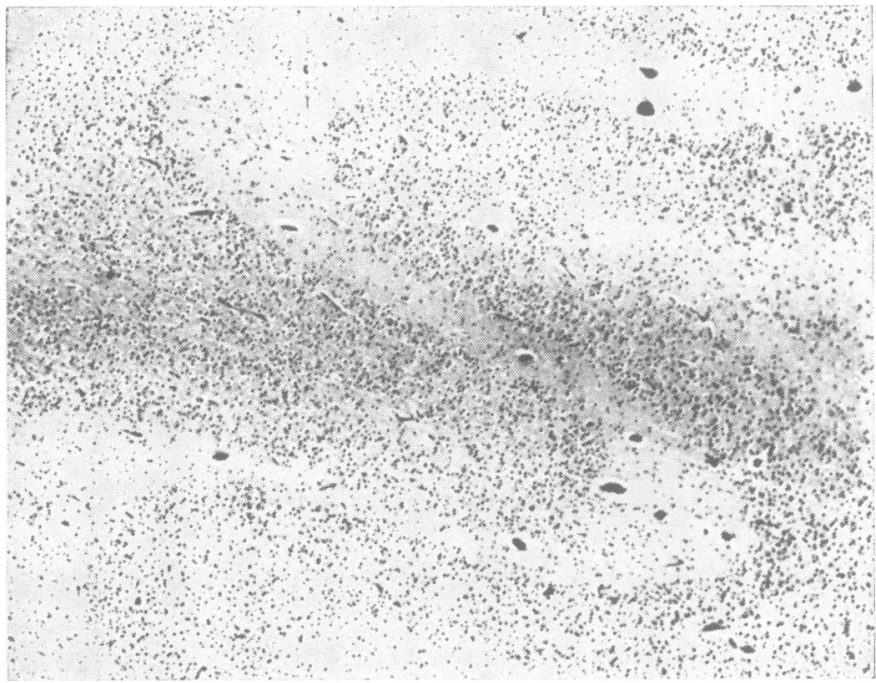

FIG. 4. Low power micrograph of polymicrogyria. Glandular pattern of the gyri and apparent fusion of the 'molecular layers' is illustrated. $H$ and $E, \times 30$.

gyric pattern replaced the normal pallium which was about twice the thickness of the abnormal cortex. The superficial layer was relatively acellular and resembled the molecular layer of normal cerebrum. Mainly tiny spindle-shaped cells were seen at this level. There was apparent fusion of adjacent 'molecular layers', so that the pia-arachnoid membrane, normally interposed between individual gyri, was not seen. The second layer, only three to four cells thick, was comprised of small dark granular neurones which were closely packed together and often grouped in clusters. Similar granular cells, more widely separated from each other, and occasional larger pyramidal cells were the components of the innermost layer, which by comparison was relatively thick. The ${ }^{\omega}$ underlying white matter was pale and stained poorly for. myelin. No cerebral heterotopiae were present. In tbe cerebellum the deep nuclei were normal. Innumeralse heterotopic foci of neurones were seen in the white mater near the midline. These foci consisted of a haphazand arrangement of cells from all three layers of the cerebelligr cortex. Several of them were located immediately beneath the ependymal lining of the fourth ventricle, forming smals hillocks that protruded into the ventricular cavity (Fig. No abnormalities of the red nuclei, inferior oliva nuclei, spinocerebellar tracts, cerebellar peduncles, or other parts of the brain-stem were seen.

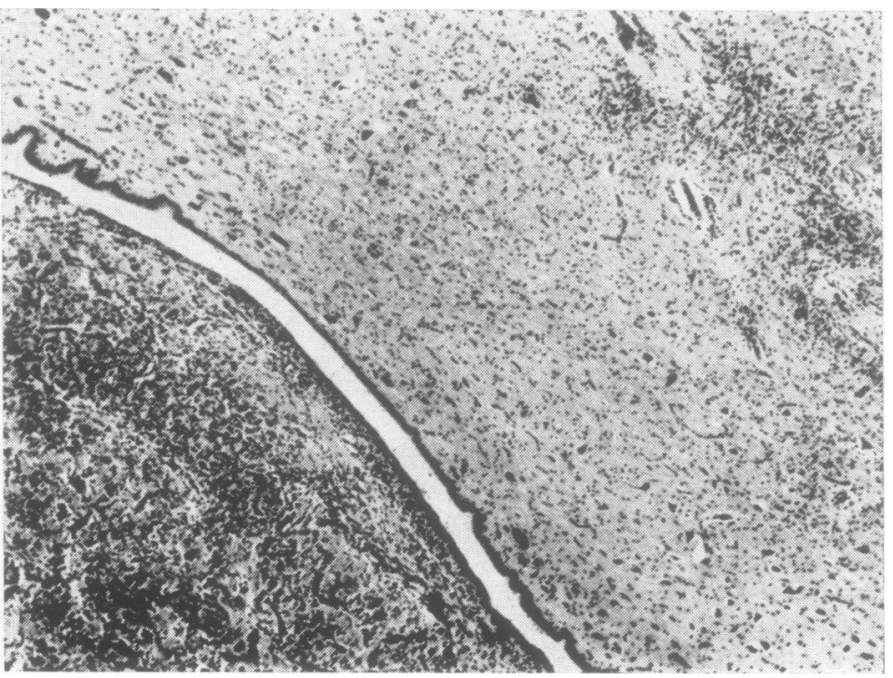

FIG. 5. Microscopic view of cerebellar heterotopia which forms a hillock protruding into fourth ventricle. $H$ and $E, \times 90$. 
CASE 2

The patient was the second born of fraternal twins whose gestational age was 38 weeks and whose intrauterine development had been uncomplicated. Their 25 year old mother had three normal children at home. The older infant who weighed $2,875 \mathrm{~g}$ and measured $49 \mathrm{~cm}$ in crown-heel length was entirely normal. After a prolonged second stage of labour lasting 40 minutes, the younger child was delivered. His birth weight was $2,749 \mathrm{~g}$ and his crown-heel length was $49 \mathrm{~cm}$. Absence of the abdominal muscles which produced massive enlargement of the abdomen was noteworthy. Both testes were undescended. In the premature nursery cystourethrography was carried out to show dilatation of the bladder and ureters. Bilateral nephrostomies were performed. On the tenth day of life the child developed an $E$. coli urinary tract infection. Although antibiotic therapy was instituted, the baby began to lose weight, his urinary output diminished, and his blood urea nitrogen began to rise. At age 23 days Proteus and Pseudomonas were cultured from the urine. The child's respirations became more feeble and cyanosis supervened. He died on the 28th day of life.

At necropsy the baby weighed $2,480 \mathrm{~g}$. The head circumference measured only $28.5 \mathrm{~cm}$, while the chest measured $33 \mathrm{~cm}$. The vertex of the skull was flat and the ears were low set. The arch of the palate was normal. A pigeon breast deformity was present. The intercostal, serratus posterior, and deep muscles of the back were normal. However, the rectus abdominis, external and internal oblique, and transversalis muscles were totally absent on gross and histological inspection. The lungs were firm, reflecting a bilateral confluent $E$. coli pneumonia. There was multicystic dysplasia of the kidneys. The ureters were massively dilated. They joined the seminal vesicles which were dilated and which entered a distended bladder at its trigone. The utriculus of the prostate was greatly enlarged, causing bladder neck obstruction. There was a patent urachus which communicated with the fundus of the bladder. The testes had remained in the abdomen overlying the ureters.

The brain and spinal cord were fixed in $10 \%$ formalin for 10 days. The brain weighed only $260 \mathrm{~g}$. There was polymicrogyria of the superior frontal gyrus, cingulate gyrus, paracentral lobule, and superior parietal lobule. Multiple coronal sections made $5 \mathrm{~mm}$ apart through the cerebral hemispheres revealed a normal ventricular system. No abnormalities of the corpus callosum or its neighbouring structures were noted. No gross lesions were apparent in serial sections of the brain-stem, cerebellum, and spinal cord. Specimens from the frontal, temporal, parietal, and occipital lobes, basal ganglia, thalamus, brain-stem, cerebellum, and spinal cord at cervical, thoracic, lumbar, and sacral levels were processed for microscopy and subjected to haematoxylin and eosin, Nissl (thionin), myelin (Weil), and Holzer stains. Microscopically a pattern of polymicrogyria similar to that found in case 1 was seen. Numerous similar foci of heterotopic grey matter were clustered near the midline of the corpus medullaris in the cerebellum. The brainstem was histologically normal. The spinal anterior horn cells were reduced in number at lower thoracic levels

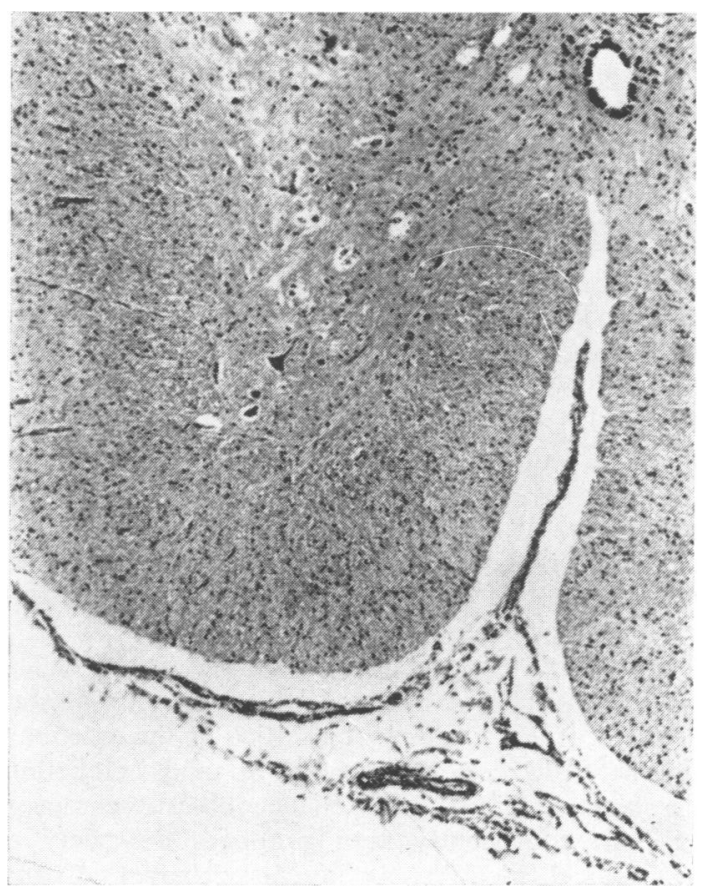

FIG. 6. Microscopic picture of anterior horn region at lower thoracic level. Marked loss of neurones is evident. $H$ and $E, \times 90$.

between T7 and T12 segments (Fig. 6). Involved anterior horn regions contained an average of 10 to 12 motor neurones, compared with the normal complement of 20 to 25 at similar levels of spinal cords from comparably aged infants in this laboratory. The remaining anterior horn cells in the affected regions were normal. No glial reaction was apparent. The anterior roots of segments T7 through T12 stained poorly for myelin.

\section{DISCUSSION}

In the earliest published case of this syndrome with neurological implications, there was presumed spinal bifida, but no radiological or neuropathological studies were done (Housden, 1934). Several references to associated meningomyelocele and hydrocephalus in the literature (Roberts, 1956; Andrén, Bjersing, and Lagergren, 1964) seem to be based on the same case described by Mathieu, Goldowsky, Chaset, and Mathieu (1953), in which there was absence of the abdominal muscles and an undescended left testis. Aplasia of ganglion cells in the bladders of two patients, linking this syndrome perhaps to Hirschsprung's disease, has been reported (Henley and Hyman, 1953). This work has not been substantiated by other investigators (Nunn and 
Stephens, 1961). Mental retardation has been found in one patient (Williams and Burkholder, 1967), although no neuropathological correlation was available.

In the present two cases, certain neuropathological features are common to both: microcephaly, microencephaly, polymicrogyria, and cerebellar heterotopiae. Moreover, in case 1 there is absence of the corpus callosum and partial agenesis of the cerebellar vermis. This combination has seldom been reported (Guttman, 1929; Lhermitte, De Ajuriaguerra, and Trotot, 1944; Svaty and Masek, 1950). Case 2 is distinguished by a reduction of anterior horn cells at lower thoracic levels. Many of the malformed structures in this syndrome are located in the midline. The predilection for the midline, reminiscent of a dysraphic state, is demonstrated in neural structures as well as bladder, prostate, abdominal wall, sternum, and palate.

Frequently polymicrogyria causes neurological symptoms and is associated with other abnormalities of the central nervous system, such as microcephaly and heterotopiae of the cerebrum and cerebellum (Crome and Stern, 1967). The cobblestone appearance so consistently seen produces a variety of histological pictures. In a classic paper, Crome (1952) describes the three-layer cortex seen in the present cases and several other microscopical patterns which may be encountered, the four-layer cortex being most common. The pathogenesis of polymicrogyria has never been definitely established. Presumably a disturbance in migration of the subependymal neuroblasts, which in the normal embryo originally reside near the ventricular system and gradually move centrifugally to form the cerebral cortex, has occurred. A similar mechanism is thought to be responsible for the commonly associated heterotopiae. Although authorities cannot agree on the precise timetable governing such migratory disturbances (Warner, 1953; Bertrand and Gruner, $1955)$, it is probable that they originate at about 56 days of intrauterine life when the cortex begins to differentiate (Millen, 1963).

Absence of the corpus callosum, first reported by Reil in 1812 (Gyepes and Gannon, 1963), has been described about 200 times (Loeser and Alvord, 1968a). As in case 1, the characteristic features include lateral displacement of the septum pellucidum and fornix, presence of Probst's bundle, which represents uncrossed callosal fibres that run in a longitudinal direction, and dilatation of the posterior horns of the lateral ventricles (Loeser and Alvord, 1968a). Polymicrogyria is commonly present and may involve the medial surfaces of the cerebral hemispheres (Menkes, Philippart, and Clark, 1964). Cerebellar heterotopiae are occasionally found (Loeser and Alvord, 1968a). Absence of the cingulate gyrus, anterior commissure, and septum pellucidum may occur (Marburg, 1949). The clinicaf? picture is quite variable and is generally due to the malformations associated with callosal absences rather than to the defect itself (Loeser and Alvord 1968a). Absence of the corpus callosum is frequently seen in conjunction with congenital malformation outside the nervous system (Loeser and Alvord: 1968a). The earliest fibres of the corpus callosum appear in the embryo at 74 days. At about 84 dayso the genu and splenium are recognizable, but noto until 115 days is the adult morphology achieved (Loeser and Albord, 1968b). Hence it is likely that the failure in development of the corpus callosum occurs between 74 and 84 days or between the $10 \mathrm{th}^{\mathrm{Cs}}$ and 12th weeks of the first trimester.

A much rarer event is absence of the cerebellarvermis, only 20 cases of which are recorded in the medical literature (Dow and Moruzzi, 1958; Joubert, Eisenring, Robb, and Andermann, 1969).믕 Several examples have also been described in animals $s_{\omega}^{\omega}$ (Dow, 1940; Verhaart, 1942; Done and Harding, 1966). The subject has recently been reviewed by Joubert et al. (1969) who reported a family weth agenesis of the cerebellar vermis accompanied by hyperpnoea, abnormal eye movements, ataxia, a\&dmental retardation. The anomaly is not usuady familial and is often without neurological symptoms. Complete absence of the vermis is much less common? than partial absence, which invariably involves the posterior inferior vermis. A related condition, ofton confused with vermal agenesis, is the DandyWalker syndrome (Dandy and Blackfan, 1914; Taggart and Walker, 1942). Dolichocephaly, higho insertion of the tentorium, and internal hydro-s cephalus with marked dilatation of the fourtho ventricle are prerequisites of this syndrome. Theö cause of the Dandy-Walker malformation is noto known, but many authorities regard the hydro- $\overrightarrow{0}$ cephalus as primary with secondary effects on the 3 vermis (Brodal and Hauglie-Hanssen, 1959). The? defect in case 1 most closely resembles that described by Sahs (1941). In both cases the anterior lobe, the flocculus, and the cerebellar hemispheres were present, although in the previously reported case in: which no anomalies outside the cerebellum were 3 . found the hemispheres were small and remnants of the posterior inferior vermis were evident. A₹ knowledge of the embryogenesis of the cerebellumo (Larsell, 1947) elucidates the pathogenesis of vermal aplasia, since the embryonic development of theo cerebellum recapitulates its phylogenetic development (Hausman, 1929; Nieuwenhuys, 1967). Theos archicerebellum, its oldest component, is comprisedn of the flocculus. This component develops earliestn 
on the phylogenetic scale, being the major or sole portion of the cerebellum in lower vertebrates such as the cyclostomes. It appears in the human embryo at 7 to 8 weeks. The paleocerebellum or anterior lobe, made up of the lingula, central lobule, culmen, and anterior quadrangular lobule, represents that part of the cerebellum receiving the spinocerebellar tracts and is most prominent in fish and reptiles. In the embryo of man the anterior lobe develops by the 11 th week. The posterior inferior vermis and the hemispheres, the neocerebellum, reach their greatest development in primates. These structures are not fully formed until nearly 15 weeks in man. A malformation of the posterior inferior vermis as seen in case 1 probably occurred during the latter first trimester.

The spinal cord from a case of absent abdominal muscles was examined at necropsy by Lichtenstein (1939) and, as in case 2, was found to contain a decreased population of anterior horn cells in the thoracic area. Since this change was confined to the same level of the cord that innervated the muscles of the abdomen, Lichtenstein considered it retrograde neuronal loss reflecting the absence of muscles. This interpretation may be valid for the present case as well. Alternatively, one cannot exclude the possibility that certain anterior horn neurones were congenitally absent, causing instead failure of muscular development in the abdominal region. Such a condition would occur during the time of embryonic anterior horn cell formation at approximately 27 days of foetal life (Millen, 1963). The concept of motor neurone agenesis is well established. Möbius's syndrome, or congenital facial diplegia, is commonly considered the product of nuclear aplasia (Henderson, 1939). Some cases of arthrogryposis multiplex congenita may be due to hypoplasia of the motor neurone system of the spinal cord (Drachman, 1968). In both Möbius's syndrome (Henderson, 1939; Evans, 1955) and arthrogryposis multiplex congenita (Drachman, 1968) club foot is frequently found. Although pathological evaluation is as yet incomplete, some authors attribute the deformity in many cases to anterior horn cell disease (Drachman, 1968). The finding of talipes equinovarus in case 1 is thus intriguing, but unfortunately permission to study the spinal cord and peripheral structures was not obtained.

Since the syndrome of absent abdominal muscles was first described, investigators have argued about its pathogenesis. Because distal urinary tract obstruction has been present in nearly $50 \%$ of cases in some series (Bourne and Cerny, 1967), some workers have theorized that obstruction early in foetal development, producing massive dilatation of the bladder, interfered with the formation of the abdominal muscles (Greene, Emmett, Culp, and Kennedy, 1952; Lattimer, 1958). However, this proposal does not explain the urogenital and abdominal defects in the cases without urinary tract obstruction, nor does it explain the frequently observed congenital anomalies unassociated with the urinary tract. Furthermore, it ignores those numerous cases of congenital urinary tract obstruction with megalobladder and megaloureter which are not accompanied by absence of abdominal muscles (Eagle and Barrett, 1950). Nunn and Stephens (1961) believe that this syndrome is the result of faulty embryogenesis rather than mechanical obstruction. They contend that the basic problem arises from deranged muscularization of the bladder, ureteral, and abdominal walls occurring between 6 and 10 weeks of foetal life. Those cases in which polycystic dysplasia of the kidneys is found support such a theory, since the crucial development of the metanephros occurs from 4 to 8 weeks (Arey, 1965). The neuropathological findings in the two cases presented here suggests that this syndrome is, indeed, a developmental defect involving many organ systems and that whatever influence produced the defects was present during the first trimester.

\section{REFERENCES}

Andrén, L., Bjersing, L., and Lagergren, J. (1964). Congenital aplasia of the abdominal muscles with urogenital malformations. Acta radiol. Diagn. (Stockh.), 2, 298-304.

Arey, L. B. (1965). Developmental Anatomy, 7th edition, pp. 301-308. W. B. Saunders: Philadelphia.

Bertrand, I., and Gruner, J. (1955). The status verrucosus of the cerebral cortex. J. Neuropath. exp. Neurol., 14, 331-347.

Bourne, C. W., and Cerny, J. C. (1967). Congenital absence of abdominal muscles: report of 6 cases. J. Urol. (Baltimore), 98, 252-259.

Brierre, J. T. jun. (1963). Congenital abnormalities of the genitourinary tract: abdominal muscle dysplasia and choanal atresia. Pediatrics, 31, 290-296.

Brodal, A., and Hauglie-Hanssen, E. (1959). Congenital hydrocephalus with defective development of the cerebellar vermis (Dandy-Walker syndrome). Clinical and anatomical findings in two cases with particular reference to the socalled atresia of the foramina of Magendie and Luschka. J. Neurol. Neurosurg. Psychiat., 22, 99-108.

Crome, L. (1952). Microgyria. J. Path. Bact., 64, 479-495.

Crome, L., and Stern, J. (1967). The Pathology of Mental Retardation, pp. 109-110. J. and A. Churchill: London.

Dandy, W. E., and Blackfan, K. D. (1914). Internal hydrocephalus, an experimental, clinical, and pathological study. Amer. J. Dis. Child., 8, 406-482.

Done, J. T., and Harding, J. D. J. (1966). The relationship of maternal swine fever infection to cerebellar hypoplasia in piglets. Proc. roy. Soc. Med., 59, 1083-1084.

Dow, R. S. (1940). Partial agenesis of the cerebellum in dogs. J. comp. Neurol., 72, 569-586.

Dow, R. S., and Moruzzi, G. (1958). The Physiology and Pathology of the Cerebellum, pp. 416-444. University of Minnesota Press: Minneapolis.

Drachman, D. B. (1968). Congenital deformities produced by neuromuscular disorders of the developing embryo, pp. 
112-121 in: Motor Neuron Diseases. Contemporary Neurology Symposia, Vol. II. Edited by F. H. Norris and L. T. Kurland. Grune and Stratton: New York.

Eagle, J. F., jun. and Barrett, G. S. (1950). Congenital deficiency of abdominal musculature with associated genitourinary abnormalities: a syndrome. Report of 9 cases. Pediatrics, 6, 721-736.

Evans, P. R. (1955). Nuclear agenesis. Möbius' syndrome: The congenital facial diplegia syndrome. Arch. Dis. Childh., 30, 237-243.

Greene, L. F., Emmett, J. L., Culp, O. S., and Kennedy, R. L. J. (1952). Urologic abnormalities associated with congenital absence or deficiency of abdominal musculature. J. Urol. (Baltimore), 68, 217-229.

Guttman, L. (1929). Über einen Fall von Entwicklungsstörung des Gross- und Kleinhirns mit Balkenmangel. Psychiat.-neurol. Wschr., 31, 453-455.

Gyepes, M. T., and Gannon, W. E. (1963). Agenesis of corpus callosum. N.Y. St. J. Med., 63, 1385-1387.

Hausman, L. (1929). The comparative morphology of the cerebellar vermis, the cerebellar nuclei, and the vestibular mass. Experimental investigations. Ass. Res. nerv. Dis. Proc., 6, 193-237.

Henderson, J. L. (1939). The congenital facial diplegia syndrome: Clinical features, pathology, and aetiology; A review of sixty-one cases. Brain, 62, 381-403.

Henley, W. L., and Hyman, A. (1953). Absent abdominal musculature, genitourinary anomalies and deficiency in pelvic autonomic nervous system. Amer. J. Dis. Child., 86, 795-798.

Housden, L. G. (1934). Congenital absence of the abdominal muscles. Arch. Dis. Childh., 9, 219-232.

Joubert, M., Eisenring, J.-J., Robb, J. P., and Andermann, F. (1969). Familial agenesis of the cerebellar vermis: A syndrome of episodic hyperpnea, abnormal eye movements, ataxia, and retardation. Neurology (Minneap.), 19, 813-825.

Larsell, O. (1947). The development of the cerebellum in man in relation to its comparative anatomy. J. comp. Neurol., 87, 85-129.

Lattimer, J. K. (1958). Congenital deficiency of the abdominal musculature and associated genitourinary anomalies: A report of 22 cases. J. Urol. (Baltimore), 79, 343-352.

Lhermitte, J., de Ajuriaguerra, J., and Trotot, R. P. (1944). Oxycéphalie avec agénésie de la commissure calleuse et du vermis inférieur. Rév. neurol., 76, 146-147.

Lichtenstein, B. W. (1939). Congenital absence of the abdominal musculature. Associated changes in the genitourinary tract and in the spinal cord. Amer. J. Dis. Child., 58, 339-348.

Loeser, J. D., and Alvord, E. C. (1968a). Clinicopathologic correlations in agenesis of the corpus callosum. Neurology (Minneap.), 18, 745-756.
Loeser, J. D., and Alvord, E. C., jun. (1968b). Agenesis of the corpus callosum. Brain, 91, 553-570.

Marburg, O. (1949). So-called agenesia of the corpus callosum 6 (callosal defect) anterior cerebral dysraphism. Arch. Neurol. Psychiat. (Chic.), 61, 297-312.

Mathieu, B. J., Goldowsky, S., Chaset, N., and Mathieu, P. L., jun. (1953). Congenital deficiency of the abdominal $\Omega$ muscles (with associated multiple anomalies). J. Pediat., 42, 92-98.

Menkes, J. H., Philippart, H., and Clark, D. B. (1964). Hereditary partial agenesis of corpus callosum. Biochemical and pathological studies. Arch. Neurol. (Chic.), 11, 198-208.

Metrick, S., Brown, R. H., and Rosenblum, A. (1957). Congenital absence of the abdominal musculature and associated anomalies. Review of recent literature and four new cases. Pediatrics, 19, 1043-1052.

Millen, J. W. (1963). Timing of human congenital malformations with a time-table of human development. is Develop. Med. Child Neurol., 5, 343-350.

Nieuwenhuys, R. (1967). Comparative anatomy of the cerebellum. Progr. Brain Res., 25, 1-93.

Nunn, I. N., and Stephens, F. D. (1961). The triad syndrome: A composite anomaly of the abdominal wall, urinary system, and testes. J. Urol. (Baltimore), 86, 782-794.

Parker, R. W. (1895). Case of an infant in whom some of the abdominal muscles were absent. Trans. clin. Soc. Lond., $\omega$ 28, 201-203.

Roberts, P. (1956). Congenital absence of the abdominal muscles with associated abnormalities of the genito urinary tract. Arch. Dis. Childh., 31, 236-239.

Sahs, A. L. (1941). Congenital anomaly of the cerebella vermis. Arch. Path., 32, 52-63.

Silverman, F. N and Huang, N. (1950). Congenital absence of the abdominal muscles associated with malformatio $\mathbb{Q}$ of the genitourinary and alimentary tracts; report of case and review of literature. Amer. J. Dis. Child., 80, 91-12年

Svaty, J., and Masek, R. (1950). Agenesia of corpus callosum and septum pellucidum with porencephaly of cerebellum Căs. Lék. Cěs., 89, 1171-1177

Taggart, J. K., Jr., and Walker, A. E. (1942). Congenital atresia of the foramens of Luschka and Magendie. Arch. Neurol. Psychiat. (Chic.), 48, 583-612.

Verhaart, W. J. C. (1942). Partial agenesis of the cerebellum and medulla and total agenesis of the corpus callosum in a goat. J. comp. Neurol., 77, 49-60.

Warner, F. J. (1953). The histogenic principle of microgyria and related cerebral malformations. J. nerv. ment. Dis., 118, 1-18.

Williams, D. I., and Burkholder, G. V. (1967). The prune belly syndrome. J. Urol. (Baltimore), 98, 244-251. 